\title{
NASA-CR-204673 Modeling of Jovian hectometric radiation source locations: Ulysses observations
}

\author{
J. D. Menietti \\ Department of Physics and Astronomy, University of Iowa, lowa City \\ M. J. Reiner \\ Hughes STX. Lanham, Maryland
}

\begin{abstract}
The Unified Radio and Plasma Wave (URAP) experiment on Ulysses has provided unique high latitude measurements of Jovian hectometric radiation (HOM) during its encounter with Jupiter in February 1992. URAP was the first radio instrument in the Jovian environment with radio direction-finding capability, which was previously used to determine the HOM source locations in the Jovian magnetosphere. These initial source location determinations were based on several assumptions, including the neglect of refractive effects, which may be tested. We have, for the first time, combined the measured incident ray-direction at the spacecraft with a model magnetosphere to directly trace the rays back to the HOM source. We concentrate on the observations of HOM from high northern latitudes when Ulysses was at distances $<15 R_{J}$. The threedimensional ray-tracing calculations presented here indicate that the HOM sources probably lie on $L$ shells in the range $3 \leq L<7$ (tilted dipole magnetic field model) consistent with previous determinations that ignored the effects of refraction. The raytracing results, however, indicate that wave refraction due to the lo torus and the magnetic field can significantly influence the precise source location. We show that constraints on the locations imposed by the gyroemission mechanism suggest that the lo torus density may have experienced temporal and/or spatial fluctuations during the Ulysses observations of HOM. Finally, in the cold plasma approximation we demonstrate that even if the emission were nearly linearly polarized near the source region, almost circular polarization will be observed at Ulysses, in agreement with observations.
\end{abstract}

\section{Introduction}

Intense hectometric radio emission (HOM) is observed at Jupiter and lies in the frequency range from perhaps $100 \mathrm{kHz}$ to over $2 \mathrm{MHz}$ [cf. Carr et al., 1983]. At the higher frequencies it is difficult to distinguish from decametric (DAM) radio emission, which has been well studied. In fact, recent observations of Jovian emissions made by the Wind satellite suggest that perhaps much of HOM is a low-frequency extension of DAM (M. L. Kaiser, personal communication, 1996). HOM is most probably RX mode emission generated by the cyclotron maser instability.

The source location of HOM has been controversial for many years. Calvert [1983] analyzed the Faraday rotation of the HOM radio emission observed by Voyager 1 near closest approach and concluded that this radio source was located near the gyrosurface and along $L=6$ (the Io flux tube). Ladreiter and Leblanc [1989, 1990a, b] have reported ray-tracing studies of HOM observed by the Voyager tlybys of Jupiter. Ladreiter and Leblanc [1990a] argued that ray-tracing calculations, performed with large wave normal angles $\left(70^{\circ}-90^{\circ}\right)$ as suggested by the cyclotron maser instability mechanism, indicate emission sources located along field lines in the range of $L$ shells $15<L<20$ (Jovian auroral zone). From these source locations, the ray paths to the Voyager spacecraft (located near

Copyright 1996 by the American Geophysical Union

Paper number 96.JA02403.

$(1148-(0227 / 96 / 96 \mathrm{JA}-(12403 \$ 309.00)$ the Jovian equatorial plane) will he significantly refracted by the Io plasma torus, as a function of frequency, resulting in an emission shadow zone that may extend radially $20 R$, or more. Since Ladreiter and Leblanc had no a priori knowledge of the direction of propagation of the incident radiation, they considered only rays in the meridian plane propagating parallel to the magnetic equator. For ray propagation directed out of the meridian plane, the source locations may be significantly altered.

Reiner et $a l$. [1993a, b] have reported the direct determination of HOM source locations based on direction of arrival measurements made by Ulysses during the Jupiter flyby. In contrast to Ladreiter and Leblanc [1989, 1990a, b], knowledge of the incident direction of the radiation provides a direct, clearly defined constraint on the possible locations of the HOM sources in the Jovian magnetosphere, without the necessity of introducing constraints from the emission mechanism.

The Ulysses HOM direction-finding results were used to determine HOM source locations for two different observational geometries. Reiner et al. [1993b] reported the determination of HOM sources detected when Ulysses was near the equatorial plane at distances $r>40 R_{J}$. These results indicated both northern and southern hemisphere HOM sources all located on low $L$ shells, $4<L<6$. Reiner et al., [1993a] reported observations of HOM made while Ulysses was located at $r<14 R$, and at Jovigraphic latitudes $>30^{\circ}$. In this work the HOM sources were again found to be located at low $L$ shells along field lines that could pass through the Io torus. In subsequent studies, Ladreiter et al. [1994], using a slightly 


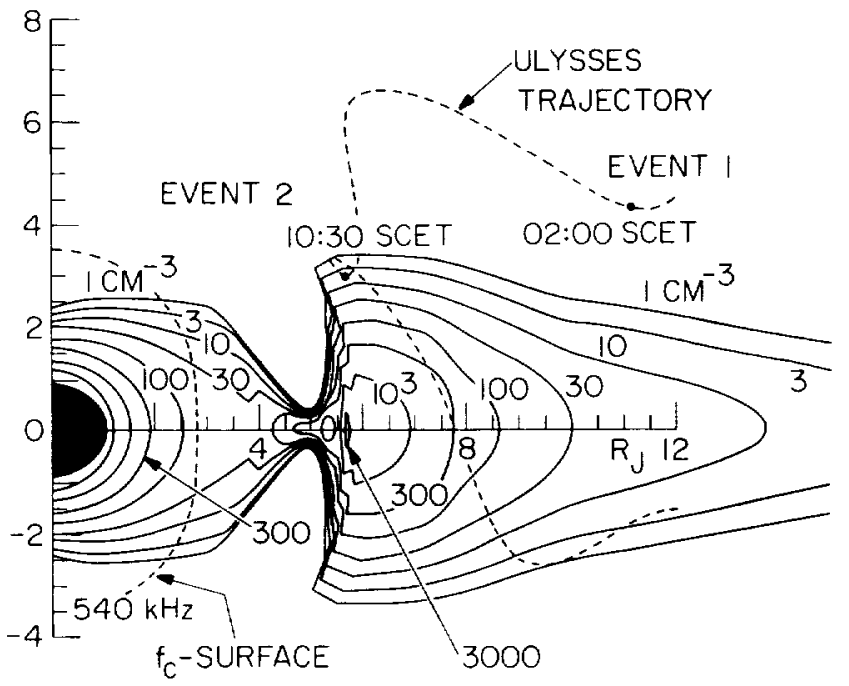

Figure 1. A projection of the Ulysses spacecraft trajectory superimposed on contours of the Io plasma torus density as determined from the model by Divine and Garrett [1983]. The locations of events 1 and 2 are indicated.

different method of analysis, found auroral region sources for HOM in the range $7<L<11$. For all of these analyses, straight-line propagation of the radiation was assumed, and possible refractive effects were ignored.

In this paper we have combined the measured incoming wave direction with ray tracing in a model Jovian magnetosphere. The model magnetosphere combines the densities [Divine and Garrett, 1983] with the O6 magnetic field model [Connerney, 1993]. The location of the radio source is determined by tracing the rays from the spacecraft back along the direction of the incident radiation to the $\mathrm{RX}$ cutoff. The results of this paper are consistent with HOM sources located at low $L$ shells but also indicate that the Divine-Garrett time-independent model may not be completely adequate to account for the HOM source directions observed during the February 1992 Ulysses encounter.

\section{Model}

The ray-tracing code is based on the Haselgrove [1955] equations and has been discussed in the past [cf. Menietti et al., 1987]. For the Jovian magnetosphere we have incorporated the analytical plasma density model of Divine and Garrett [1983], and the O6 magnetic field model of Connerney [1993]. The O6 magnetic field model is a fit to the Pioneer 11 and Voyager observations using a spherical harmonic expansion and is also a static model. This model is an improvement over the $\mathrm{O} 4$ model [Acuna and Ness, 1976] as discussed by Connemey [1993].

The Divine-Garrett plasma torus model is a threedimensional (3-D), static, analytical, and empirical model with azimuthal symmetry and is based on the Pioneer and Voyager flyby data. It incorporates the Io plasma torus densities first observed in detail by the Voyager spacecraft [Bagenal et al., 1985; Bagenal and Sullivan, 1981]. It should be noted that no direct measurements of plasma have been made near the planet $\left(r<4 R_{J}\right)$, and the Divine-Garrett model in this region is an extrapolation. The torus is believed to consist of separate cold and warm regions, and the boundary between these regions extends to perhaps $3 R$, along magnetic field lines. This empirical model was used because it lends itself well to raytracing calculations and agrees reasonably well (within a factor of -2) to the most recently published tabular model [Bagenal, 1994]. In Figure 1 we display contours of the plasma density of the Io torus determined from the Divine and Garrett [1983] model (modified version of their Figure 11).

It is becoming increasingly clear, however, that torus models based on the Voyager flyby observations may not adequately account for spatial and temporal variations of plasma density. Desch et al. [1994], for instance, conclude that significant azimuthal asymmetries (namely, $30-50 \%$ density depletions) are necessary to explain Ulysses radio observations in the high latitude Io plasma torus. Schneider and Spencer [1991] have obtained ground-based observations indicating strong longitudinal and temporal dependences associated with the Io torus, which no doubt influence radio emissions that are refracted by the high density regions.

The ray-tracing code requires as input the initial wavenormal angle $\left(\psi_{0}\right)$ and the azimuthal angle, $\beta_{0}$, of the $k$ vector about the magnetic field direction. These angles were derived directly from the Ulysses observations at the spacecraft as opposed to the HOM source point. The measured direction angles at Ulysses, which define the direction of the ray wave vector $\mathbf{k}$ at the spacceraft, were first transformed from the spacecraft frame to Jovian System III coordinates. To determine the wave normal angle of the ray from these direction angles, a transformation was made to a coordinate system directed along the $B$ field vector direction at Ulysses. The wave direction angles at Ulysses are defined in this system where $\psi$ is the angle between $\mathbf{B}$ and $\mathbf{k}$ (the wave normal angle) and $\beta$ is the azimuthal angle of the projection of $\mathbf{k}$ on the plane perpendicular to $\mathbf{B}$.

The direction-finding capability makes use of a synthesis of signals from the spin plane (72.5 $\mathrm{m}$ tip-to-tip dipole) and spin axis ( $7.5 \mathrm{~m}$ monopole) antennas. These data provide the arrival direction and complete polarization state (four Stokes parameters) of the radiation [Manning and Fainberg, 1980]. The two angles that identify the direction of the incoming HOM radiation were obtained from the analysis of the resulting spin modulated signals from the radio receivers observed for each 12-s spacecraft spin period. The sampling rate is frequency dependent. Variations of the source intensity over the 12-s measurement cycle, as well as errors due to receiver noise and quantization errors in the telemetry, lead to a significant scatter in the individual angle determinations [e.g., Reiner et al., 1993a, Figure 2]. To reduce these crrors, the source directions used in the ray tracing were obtained by averaging the individual direction measurements over a period of about $30 \mathrm{~min}$. This averaging introduces errors duc to the motions of the spacecraft and the source in the time over which measurements were made, but these latter errors are estimated to be significantly smaller than those due to the above effects. Finally, there may be systematic errors in these measured angles due to the calibration of the receiver/antenna system. To calibrate, we used the observations of HOM in the same frequency range measured when Ulysses was far from Jupiter $\left(>50 R_{J}\right)$ to determine the receiver gain and electrical tilt angles of the monopole spin axis antenna. We estimate that this should allow us to determine the sourec angles to $-0.5^{\circ}$ in the spacecraft system.

The rays at each frequency were launched from the location of Ulysses at the measured direction angles back toward Jupiter and followed at least until the ray coordinates reached a 
Table 1. Observed HOM Emission Angles and Deduced Source Locations

\begin{tabular}{|c|c|c|c|c|c|c|}
\hline \multirow[b]{2}{*}{$\begin{array}{c}\text { Frequency } \\
\text { kHz }\end{array}$} & \multicolumn{2}{|c|}{ Direction Angles } & \multirow[b]{2}{*}{$\begin{array}{c}L \text { Shell } \\
\text { (Dipole) }\end{array}$} & \multicolumn{3}{|c|}{ Source Location } \\
\hline & $\begin{array}{l}\psi_{0} \\
\text { deg }\end{array}$ & $\begin{array}{l}\beta_{i,} \\
\text { deg }\end{array}$ & & $r\left(R_{J}\right)$ & $\begin{array}{c}\lambda, \\
\operatorname{deg}\end{array}$ & $\begin{array}{l}\Lambda_{111}, \\
\text { deg }\end{array}$ \\
\hline \multicolumn{7}{|c|}{ Event I } \\
\hline 540 & $153.8 \pm 2$ & $262.0 \pm 4$ & $5.3 \pm 0.2$ & 3.20 & 39.0 & 49 \\
\hline 940 & $151.6 \pm 2$ & $270.0 \pm 4$ & $3.9 \pm 0.1$ & 2.62 & 35.0 & 34 \\
\hline \multicolumn{7}{|c|}{ Event 2} \\
\hline 540 & $153.4 \pm 2$ & $217.1 \pm 4$ & $7.2 \pm 0.2$ & 3.30 & 47.4 & 105 \\
\hline 740 & $151.4 \pm 2$ & $223.8 \pm 4$ & $6.4 \pm 0.3$ & 2.97 & 47.1 & 116 \\
\hline
\end{tabular}

No refraction [cf. Reiner et al., 1993a].

point ("source point") where the wave frequency was near the local RX cutoff frequency, $f_{\mathrm{RX}}=f_{\mathrm{g}} / 2+\sqrt{\left(f_{\mathrm{p}}\right)^{2}+\left(f_{\mathrm{g}} / 2\right)^{2}}$, where $f_{\mathrm{g}}$ is the gyrofrequency and $f_{\mathrm{p}}$ is the plasma frequency. As a practical criterion, we considered the source position to be any point within $5 \%$ of the local $\mathrm{RX}$ cutoff frequency.

\section{Results}

We have concentrated on events 1 and 2 as presented by Reiner et al. [1993a]. For these events, Ulysses was located at a distance of about $12 R_{J}$ and $6 R_{J}$ from Jupiter, respectively, and at latitudes $>30^{\circ}$. Event 1 occurred on February 8, 1992, from about 0100 to 0300 spacecraft event time (SCET) when Ulysses was at system III longitude, $\Lambda_{111} \sim 31^{\circ}$, and latitude, $\lambda_{\mathrm{IU}} \sim 31^{\circ}$, while event 2 occurred from about 1000 to 1130 SCET while Ulysses was at $\Lambda_{111} \sim 41^{\circ}$ and $\lambda_{111} \sim 37^{\circ}$. Specifically we have determined the wave direction angles listed in Table 1 for the SCET times 0215 (event 1) and 10:30 (Event 2). As indicated, these angles have an associated error that results primarily from uncertainties in fitting the data to the antenna equations as briefly discussed above and elsewhere [cf. Reiner et al., 1993a]. Included in Table 1 are the source positions obtained assuming no refraction of the ray [cf. Reiner et al., 1993a] for comparison with the determinations in this paper. The positions of the Ulysses spacecraft at the times of events 1 and 2 are superimposed on Figure 1.

\subsection{Event 1}

We launched a ray at the angles $\psi_{0}$ and $\beta_{0}$ given in Table 1 for $f=540 \mathrm{kHz}$, and traced this ray through the model Jovian magnetosphere toward Jupiter. Assuming that HOM emission is generated in the $\mathrm{RX}$ mode, this ray must intercept $f_{\mathrm{RX}}$ at some point along the ray path, if we have chosen the initial wave direction correctly and if the magnetospheric model is valid. In Figure 2 we display the ray path in three dimensions (Figure 2a) and in a two-dimensional projection into the $x-y$ plane (Figure $2 b$ ). Included on the plot are a portion of the spacecraft trajectory, the magnetic field line loop (O6 model) that passes through the spacecraft, and three line profiles representing planar cuts through the surface for $f_{\mathrm{RX}}=540 \mathrm{kHz}$. The star on the plot indicates the projection of the $540-\mathrm{kHz}$ RX cutoff surface at a position along the $z$ axis that corresponds to the $z$ coordinate of the ray path at the point of closest approach to Jupiter. We see that as the ray propagates toward Jupiter, it is refracted out of the meridian plane and does not reach the RX cutoff surface for $f=540 \mathrm{kHz}$ but is in fact about $0.25 R_{J}$ above this surface at the closest point

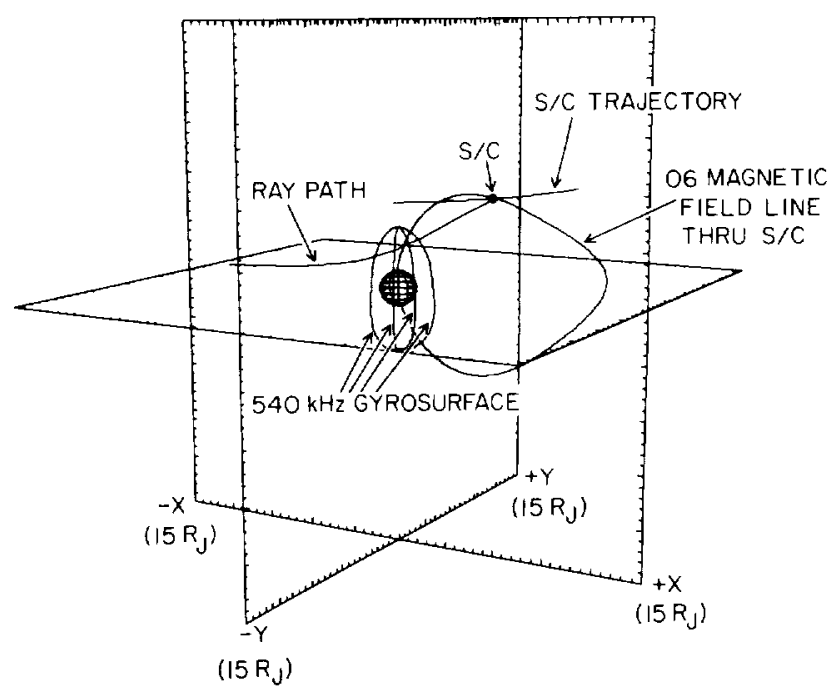

Figure 2a. Three-dimensional view, using system III coordinates, of the ray path, spacecraft trajectory, O6 field line through the spacecraft location, and planar cuts through the $540-\mathrm{kHz}$ RX cutoff surface, for the observed initial wave direction of event 1 .

$\left(f_{\mathrm{RX}}=412.5 \mathrm{kHz}\right)$. Since this particular ray refracted away from the planet just above the RX cutoff surface, $f_{\mathrm{RX}}=540$ $\mathrm{kHz}$, either the initial wave direction was in error or the magnetospheric models for plasma density and magnetic field did not accurately represent the Jovian environment at the time of the Ulysses flyby.

By slightly adjusting the launch angles at the spacecraft, however, we were able to produce a ray path that did intercept $f_{\mathrm{RX}}$ (within $5 \%$ ). This was accomplished in a systematic fashion by alternately incrementing $\psi$ then $\beta$. If $\psi$ was increased and

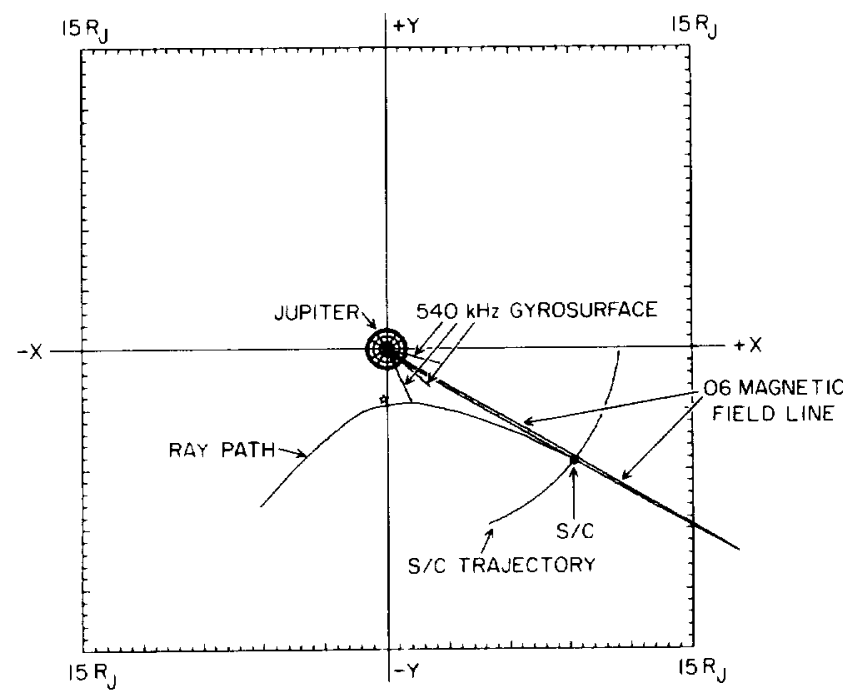

Figure 2b. View (in Jovigraphic $x-y$ plane) of the path of a ray launched at the spacecraft at $f=540 \mathrm{kHz}$ in the measured direction. Also shown are a portion of the spacecraft trajectory, the O6 magnetic field loop that intercepts the spacecraft, and three line profiles of points on the $540-\mathrm{kHz}$ RX cutoff surface. The star locates the projection of the $540-\mathrm{kHz}$ RX cutoff surface at a position along the $z$ axis where the wave ray path is closest to Jupiter. 
Table 2. Event 1 HOM Launch Angles and Calculated Source Locations for O6 Magnetic Ficld Model

\begin{tabular}{|c|c|c|c|c|c|c|c|}
\hline \multicolumn{2}{|c|}{$\begin{array}{c}\text { Direction } \\
\text { Angles }\end{array}$} & \multirow[b]{2}{*}{ L. } & \multirow[b]{2}{*}{ Torus } & \multicolumn{3}{|c|}{ Source Location } & \multirow[b]{2}{*}{$\begin{array}{l}\Delta \mathbf{k}, \\
\mathrm{deg}\end{array}$} \\
\hline $\begin{array}{l}\psi_{t} \\
\mathrm{dcg}\end{array}$ & $\begin{array}{l}\beta_{1 .} \\
\operatorname{deg}\end{array}$ & & & $\begin{array}{l}r \\
R_{J}\end{array}$ & $\begin{array}{l}\lambda \\
\operatorname{deg}\end{array}$ & $\begin{array}{l}\Lambda_{111} \\
\text { deg }\end{array}$ & \\
\hline \multicolumn{8}{|c|}{$f=540 \mathrm{kHz}$} \\
\hline 152.8 & 262.0 & $4.11^{*}$ & yes & 3.12 & .34 .6 & 72.0 & 1.68 \\
\hline 153.8 & 269.0 & $5.5 \dagger$ & yes & 3.22 & 48.8 & $48 . .3$ & 3.09 \\
\hline \multicolumn{8}{|c|}{$f=940 \mathrm{kHz}$} \\
\hline 149.9 & 269.8 & $2.3^{*}$ & yes & 2.31 & 16.1 & 38.8 & 1.68 \\
\hline 153.9 & 270.8 & $6.5 t$ & yes & 2.78 & 58.5 & 35.0 & 2.35 \\
\hline
\end{tabular}

$* L_{\text {min }}$
$\dagger L_{\text {mix }}$

the ray was found to move away from $f_{\mathrm{Rx}}$, then $\psi$ would be decreased. Then $\beta$ would be incremented in the same fashion. The ray, with initial wave direction angles at Ulysses of $\psi_{0}=$ $152.8^{\circ}$, and $\beta_{0}=265.0^{\circ}$ intercepted the "source point" where the wave frequency was approximately equal to $f_{\mathrm{R} \times}$ at $L=4.0$ (tilted dipole model). The system III latitude and longitude of the source point is tabulated in row 1 of Table 2 . Also included in Table 2 is the angular difference, $\Delta \mathbf{k}$, between the wave direction measured at Ulysses (refer to Table 1) and that used in the ray tracing. These values are generally smaller than the maximum estimated RMS error $\left(\sim 4^{\circ}\right)$ in measuring $\mathbf{k}$ (which depends on $\psi_{0}$ and $\beta_{0}$ listed in Table 1$)$. The results in row 1 of Table 2 are for a ray as close as possible to the measured direction (Table 1) that reached $f_{\mathrm{RX}}$. It was also the ray that produced the smallest value of $L\left(L_{\min }\right)$ at the source location.

To gain some idea of the range of possible source locations, we next varied values of $\psi_{01}$ and $\beta_{0}$ until we obtained a ray that intercepted the $f_{\mathrm{RX}}$ surface at a point with the largest possible value of $L$. The wave direction angles at Ulysses were $\psi_{01}=$ $153.8^{\circ}, \beta_{0\}}=269.0$ and the resulting source locations are tabulated in row 2 of Table 2 . In Figure 3 we show plots of the two rays identified in rows 1 and 2 of Table 2 . The ray paths are shown in a three-dimensional projection (Figure $3 \mathrm{a}$ ), and in the $x-y$ Jovigraphic plane (Figure 3b) as a "top" view. The two rays (one for $L_{\min }$ and one for $L_{\max }$ ) propagate out of the meridian plane but intercept the RX cutoff surface relatively near the spacecraft longitude.

Table 2 also gives the Jovian system III coordinates of the "source point" of the emission or position along the ray path where the wave frequency was within $5 \%$ of the local value of $f_{\mathrm{Rx}}$. By comparing these with the values in Table 1, we see that the source position determined from the ray tracing can differ considerably from that determined assuming no refraction and a dipole field. The range of source latitudes on the $f_{\mathrm{RX}}$ surface deduced from the ray tracing is from $34.6^{\circ}$ to $48.8^{\circ}$. The source longitude ranged from $48.3^{\circ}$ to $72.0^{\circ}$. These results indicate considerable uncertainty in the HOM source locations. The values of $39.0^{\circ}$ and $49.0^{\circ}$ from Table 1 lie within this range.

Also contained in Table 2 are the results for the ray tracing at a frequency of $940 \mathrm{kHz}$. By comparing with Table 1 we see that the values of the source position for the minimum and maximum $L$ value bracket the values of Table 1 for no refraction; and the longitudes obtained by ray tracing are also quite close to those shown in Table 1. This result is not surprising considering the smaller refractive effects expected at the higher frequency.

\subsection{Event 2}

Event 2 occurred near closest approach of Ulysses at a radial distance of $\sim 6 R_{J}$. As seen in Figure 1, this event occurred when Ulysses was near the upper edge of the torus. For event 2 , the ray corresponding to the measured wave direction angles again failed to propagate to the RX cutoff surface. To intercept the $f_{R X}$ surface, it was necessary to considerably modify the values of $\psi_{0}$ and $\beta_{0}$ from their measured values. The results of the analysis for event 2 at $f=540 \mathrm{kHz}$ and $f=740 \mathrm{kHz}$ are presented in Table 3 . The source locations for event 2 are similar to those of event 1 but generally occur at smaller longitudes than those determined assuming no-refractive effects (Table 1). We have included in row 1 of Table 3 , as in Table 2 , the results of the ray launched as close as possible to the measured direction that intercepted the RX cutoff surface. The source location for this ray (the point where $f \sim f_{\mathrm{RX}}$ ) was located at the smallest value of $L$ for all rays examined. In row 2 we include the results for the ray launched in a direction that
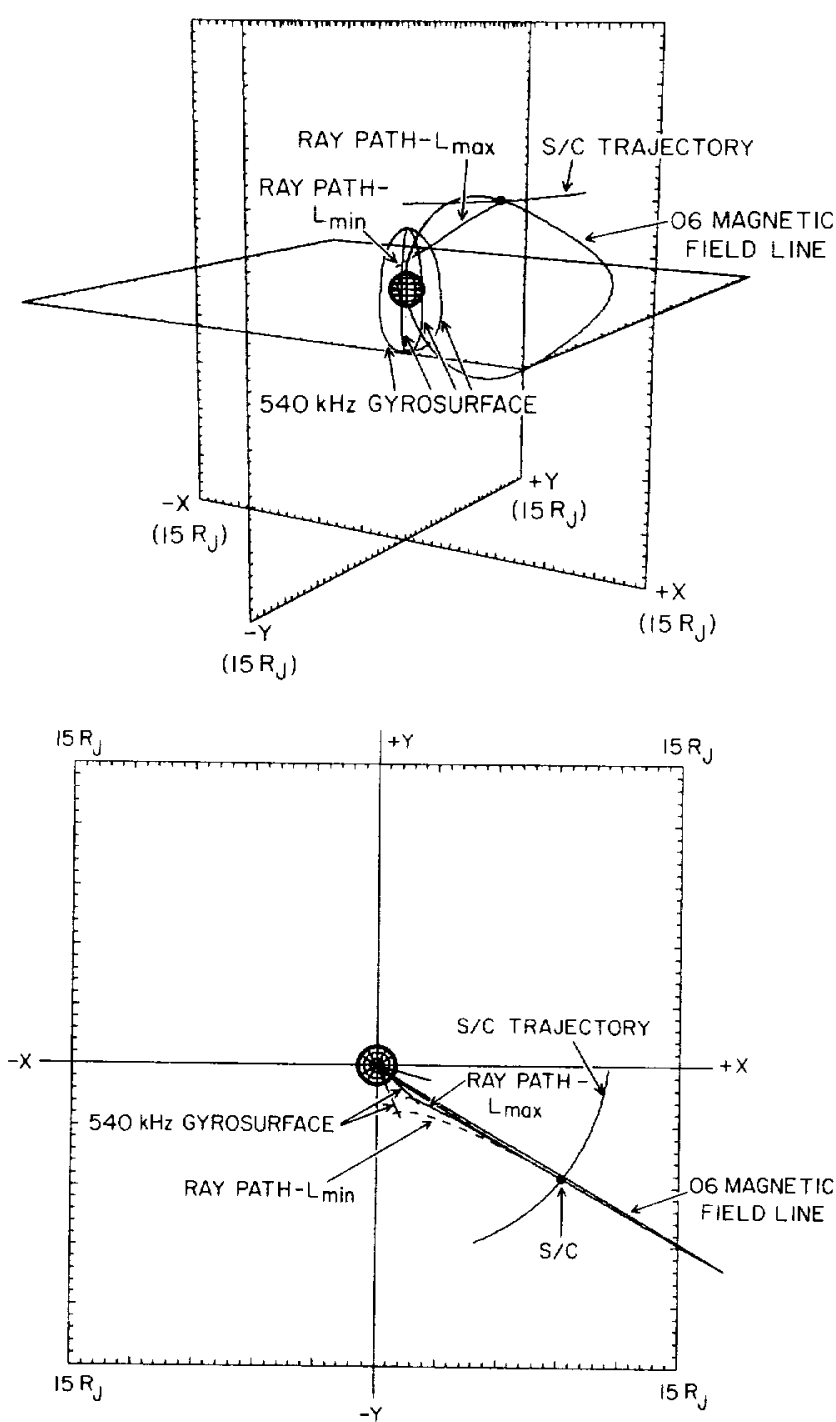

Figure 3. The format is the same as Figure 2, but now the rays launched in directions that yielded source positions at the smallest and largest possible $L$ values are shown. The ray labeled $L_{\min }$ is also the ray launched as near as possible to the observed ray direction. 
yielded a source location at the largest possible value of $L$ $\left(L_{\text {max }}\right)$. Both of these rays (rows 1 and 2 of Table 2 ) are plotted in Figure 4a (top view) and Figure 4b (3-D view).

As seen in Table 3 , the values of $\Delta \mathbf{k}$ are significantly larger than the estimated measurement uncertainty in the arrival directions of the radiation. To understand the origin of this result we analyzed the sensitivity of ray paths to modifications in the plasma density model. We have both increased and decreased the plasma torus densities by multiplying the values produced by the Divine and Garrett model by at factor ranging from 0 to 5 . We found that we could obtain a smaller value of $\Delta \mathbf{k}$ by decreasing the torus density. In Table 3 we also present the ray-tracing results obtained in the absence of a plasma torus (including only a plasmaspheric density model). In row 3 of Table 3 we indicate the results of the ray that produced not only the largest value of $L$, but also the smallest value of $\Delta \mathbf{k}$. The latitude of the source location of this ray lies intermediate between that of rows 1 and 2 in Table 3 (which were obtained including the Io torus), and the longitude is only $\sim 4$ larger. Even though the value of $\Delta \mathbf{k}$ is smaller than the results that include a torus (rows 1 and 2 of Table 3 ), it is still considerably larger than the expected maximum observational error of about $4^{\circ}$.

Another parameter in the problem is the Jovian magnetic field. In order to investigate the influence of modifications of the field model, we repeated many of the calculations at a wave frequency of $f=540 \mathrm{kHz}$ using the $\mathrm{O} 4$ magnetic field model [Actuna and Ness, 1976]. For a detailed comparison of both models, sec Comnerney [1993]. In Table 4 we present the calculations of ray tracing for both events 1 and 2 in the same format as Table 3. The source locations deduced for the $\mathrm{O} 4$ magnetic field modet are qualitatively similar to those for the O6 model. As in Tahles 2 and 3 , in row 1 of Table 4 we include the source location for the ray launched as close as possible to the measured direction, while row 2 contains the souree location at the largest possible $L$ value. For the 04 model the range of longitude values is somewhat smaller compared to the Of model, while the latitude range is larger extending to $57.5^{\circ}$ at $L_{\text {mix }}$. For event 1 the values of $\Delta \mathbf{k}$ were less than the expected measurement error.

Table 4 indicates that while the 04 model produced a smaller range of longitudes for the source region than did the Ot model, the values of $\Delta \mathbf{k}$ were still larger than the expected

Table 3. Event 2 HOM Launch Angles and Calculated Source Locations for O6 Magnetic Field Model

\begin{tabular}{|c|c|c|c|c|c|c|c|}
\hline \multicolumn{2}{|c|}{$\begin{array}{l}\text { Direction } \\
\text { Angles }\end{array}$} & \multirow[b]{2}{*}{ l. } & \multirow[b]{2}{*}{ Torus } & \multicolumn{3}{|c|}{ Source Location } & \multirow[b]{2}{*}{$\begin{array}{l}\text { Jk. } \\
\text { deg }\end{array}$} \\
\hline $\begin{array}{l}\psi_{i, 1} \\
\text { deg }\end{array}$ & $\begin{array}{l}\beta_{i n} \\
\text { deg }\end{array}$ & & & $r_{i}$ & $\begin{array}{c}\lambda \\
\operatorname{deg}\end{array}$ & $\begin{array}{l}l_{111}, \\
\text { deg }\end{array}$ & \\
\hline \multicolumn{8}{|c|}{$i=540 \mathrm{k} / \mathrm{z}$} \\
\hline 144.7 & 249.5 & 3.8 & yes & 3.10 & 31.3 & 78.3 & 18.5 \\
\hline 149.7 & 271.5 & $4.7 \dagger$ & yes & 3.13 & 44.4 & 42.9 & 25.4 \\
\hline 146.7 & 240.5 & $4.9+$ & IIO & 3.25 & 40.7 & 82.7 & 13.4 \\
\hline \multicolumn{8}{|c|}{$f=740 \mathrm{kHz}$} \\
\hline 143.8 & 255.8 & 3.2 & yes & 2.73 & 28.7 & 72.4 & 18.5 \\
\hline 150.8 & 260.8 & $5.0 \%$ & yes & 2.95 & 46.7 & 70.9 & 17.6 \\
\hline 140.8 & 248.8 & 3.3 & no & 2.76 & $30) .0$ & 77.3 & 17.3 \\
\hline 144.8 & 249.8 & $3.9+$ & no & 2.85 & 36.9 & 77.4 & 15.1 \\
\hline
\end{tabular}

"I Imin" $_{\text {. }}$

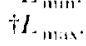
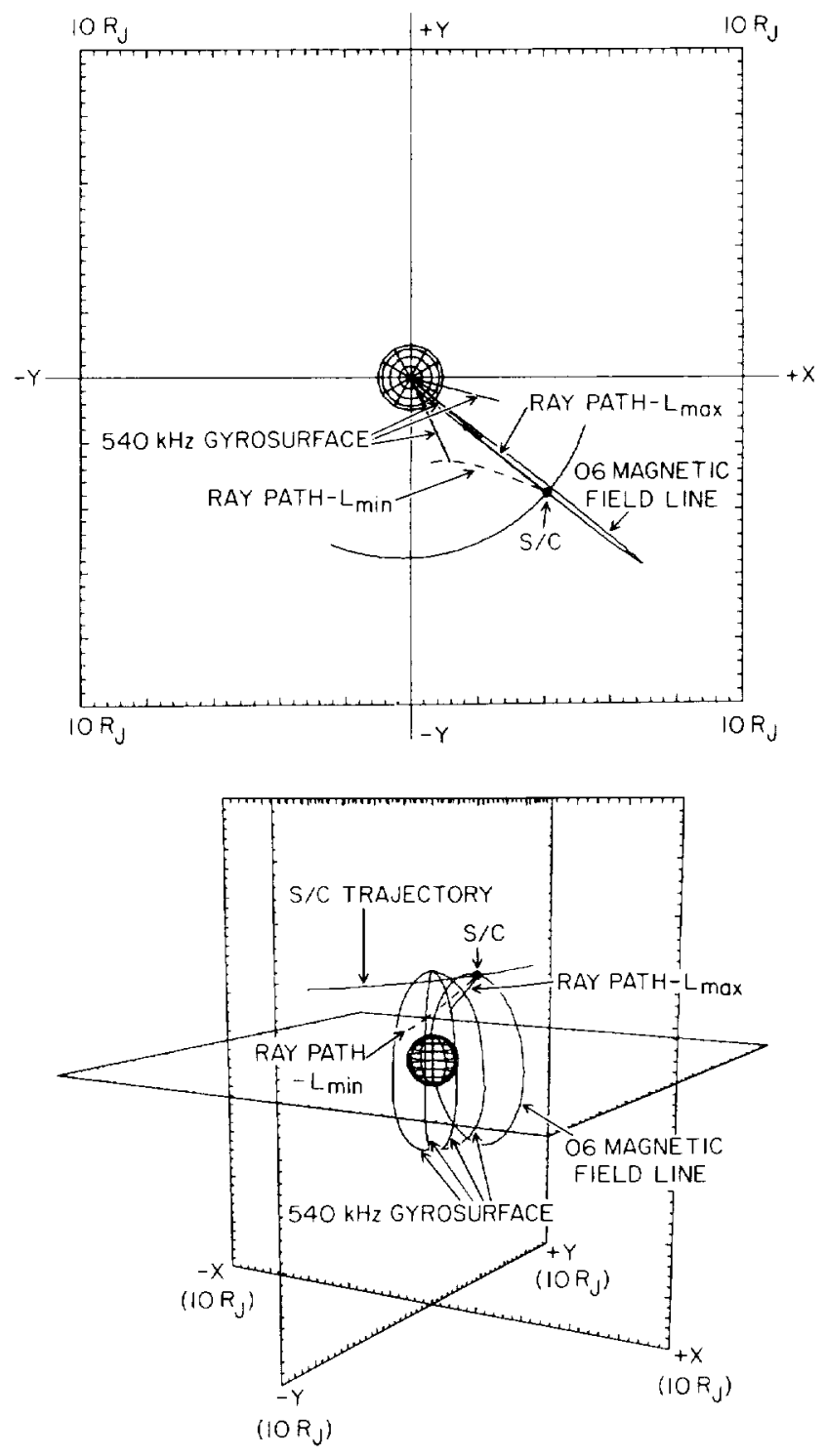

Figure 4. (a) Top and (b) three-dimensional view of the " $L_{\text {min" }}$ " and " $L_{\text {masx }}$ "rays for $f=540 \mathrm{kHz}$, when the spacecraft was at the position of event 2 . For this event there was a bigger range of possible source positions, and $\Delta \mathbf{k}$ wats larger than the expected error.

measurement error of $-4^{\circ}$. Also contained in Table 4 are the results for the case of no torus density. The range of allowable ray paths have inital wave directions that lie closer to the measured values, but $\Delta \mathbf{k}$ is still larger than $4^{\circ}$. For comparison, we show in Figure 5 sample ray paths for Event 2 using both the $\mathrm{O} 6$ and the $\mathrm{O} 4$ magnetic field model. The rays were each launched in the same direction relative to the field line. The small displacement of the $\mathrm{O} 4$ model relative to the $\mathrm{Of}$ no doubt accounts for the larger longitude values obtained in the former ray-tracing results.

\section{Polarization}

Recent observations of DAM [Dulk et al., 1994, and references therein| indicate that this emission is often elliptically polarized and that the axial ratio may depend on the viewing angle [Melrose and Dulk, 1993]. Yet Reiner et al. [1993a] re- 
Table 4. Modeled HOM Launch Angles and Source Locations for O4 Magnetic Field Model

\begin{tabular}{|c|c|c|c|c|c|c|c|}
\hline \multicolumn{2}{|c|}{$\begin{array}{c}\text { Direction } \\
\text { Angles }\end{array}$} & \multirow[b]{2}{*}{$L$} & \multirow[b]{2}{*}{ Torus } & \multicolumn{3}{|c|}{ Source Location } & \multirow[b]{2}{*}{$\begin{array}{l}\Delta \mathbf{k} \\
\operatorname{deg}\end{array}$} \\
\hline $\begin{array}{l}\psi_{1, *} \\
\operatorname{deg}\end{array}$ & $\begin{array}{l}\beta_{1 \prime} \\
\text { deg }\end{array}$ & & & $\begin{array}{l}r \\
R_{I}\end{array}$ & $\begin{array}{c}\lambda, \\
\operatorname{deg}\end{array}$ & $\begin{array}{l}\Lambda_{111}, \\
\mathrm{deg}\end{array}$ & \\
\hline \multicolumn{8}{|c|}{ Event $1: f=540 \mathrm{kHz}$} \\
\hline 151.6 & 259.1 & 3.2 & yes & 2.89 & 24.9 & 64.7 & 3.72 \\
\hline 155.1 & 263.5 & $7.5^{*}$ & yes & 3.28 & 57.5 & 43.5 & 4.01 \\
\hline \multicolumn{8}{|c|}{ Event 2: $f=540 \mathrm{kHz}$} \\
\hline 146.9 & 246.1 & 4.4 & yes & 3.10 & 37.8 & 80.3 & 16.89 \\
\hline 150.7 & 249.5 & $5.6^{*}$ & yes & 3.20 & 46.5 & 77.1 & 17.34 \\
\hline 146.9 & 237.1 & 5.2 & no & 3.18 & 43.6 & 83.5 & 12.39 \\
\hline 150.7 & 239.5 & $6.4^{*}$ & no & 3.26 & 50.0 & 79.3 & 12.53 \\
\hline
\end{tabular}

ported a high degree of right-hand circular polarization for the Ulysses observations of HOM (compare their Figure 2), i.e., they found no evidence for significant elliptical polarization. Wave polarization can be easily obtained in the cold plasma theory of wave propagation. Stix $[1962$, p. 42] defines the electromagnetic polarization as

$$
P=i E_{x} / E_{y}=\left(n^{2}-S\right) / D
$$

where $E_{x}$ and $E_{v}$ are the wave transverse electric field components, $n$ is the index of refraction, $S=(R+L) / 2$ and $D=$ $(R-L) / 2 . R$ and $L$ are defined in terms of the wave real frequency, plasma frequency, and gyrofrequency [Stix, 1962, equations $19-21$, p. 7]. We have calculated the polarization of the wave as a function of ray path. A typical example is presented in Figure 6. Here we see that $P \ll 1$ (near linear polarization) near the source point, but increases rapidly as the wave propagates outward, toward the spacecraft. Thus the observation of large ( $>90 \%$ circular) polarization is expected in the cold plasma limit, consistent with the Ulysses HOM observations. This result is seen to follow naturally from a consideration of the wave characteristics along the ray path. Near the source region the wave frequency $f \sim f_{\mathrm{Rx}}$ and the calculated wave normal angle, $\psi \sim 90^{\circ}$ as expected. As the ray propagates outward the index of refraction decreases, and $\psi$ also decreases to values $\ll 90^{\circ}$. Let us consider the two special cases, therefore, of propagation at wave normal angles near zero and near $90^{\circ}$. In the cold plasma limit it is well known for extraordinary waves with $\psi-0$,

$$
n^{2} \sim R
$$

while for $\psi \sim 90^{\circ}$,

$$
n^{2} \sim R L / S
$$

Thus, for $\psi \sim 0$

$$
P \sim(R-S) / D \sim 1
$$

while for $\psi \sim 90^{\circ}$,

$$
P=(R L / S-S) /(R-L) / 2=(L-R) /(L+R)
$$

Near the HOM source region, $f \sim f_{\mathrm{RX}}$ and $\psi \sim 90^{\circ}$, so $P<1$ as we observe in the calculations, while near the spacecraft, $\psi$ $\sim 150^{\circ}$ and $P \sim 1$ as observed. These results are not inconsistent with past observations of elliptical polarization of decametric emission for which propagation effects can be different.

\section{Discussion}

For event 1 the source locations determined from the ray tracing differed somewhat from those obtained in the study of Reiner et al. [1993a] (which assumed no refraction). For instance, at $f=540 \mathrm{kHz}$, the calculated latitude of the ray with the smallest $L$ value and the latitude of the ray with the largest $L$ value bracket the latitude of the source obtained by Reiner et al. For event 1 , if the waves were launched from Ulysses within the estimated error limit $\left(\sim 4^{\circ}\right)$ of the observed wave direction angle, the rays intercepted $f_{\mathrm{RX}}$, as required for $\mathrm{RX}$ mode emission. This was not the case for event 2 where we had
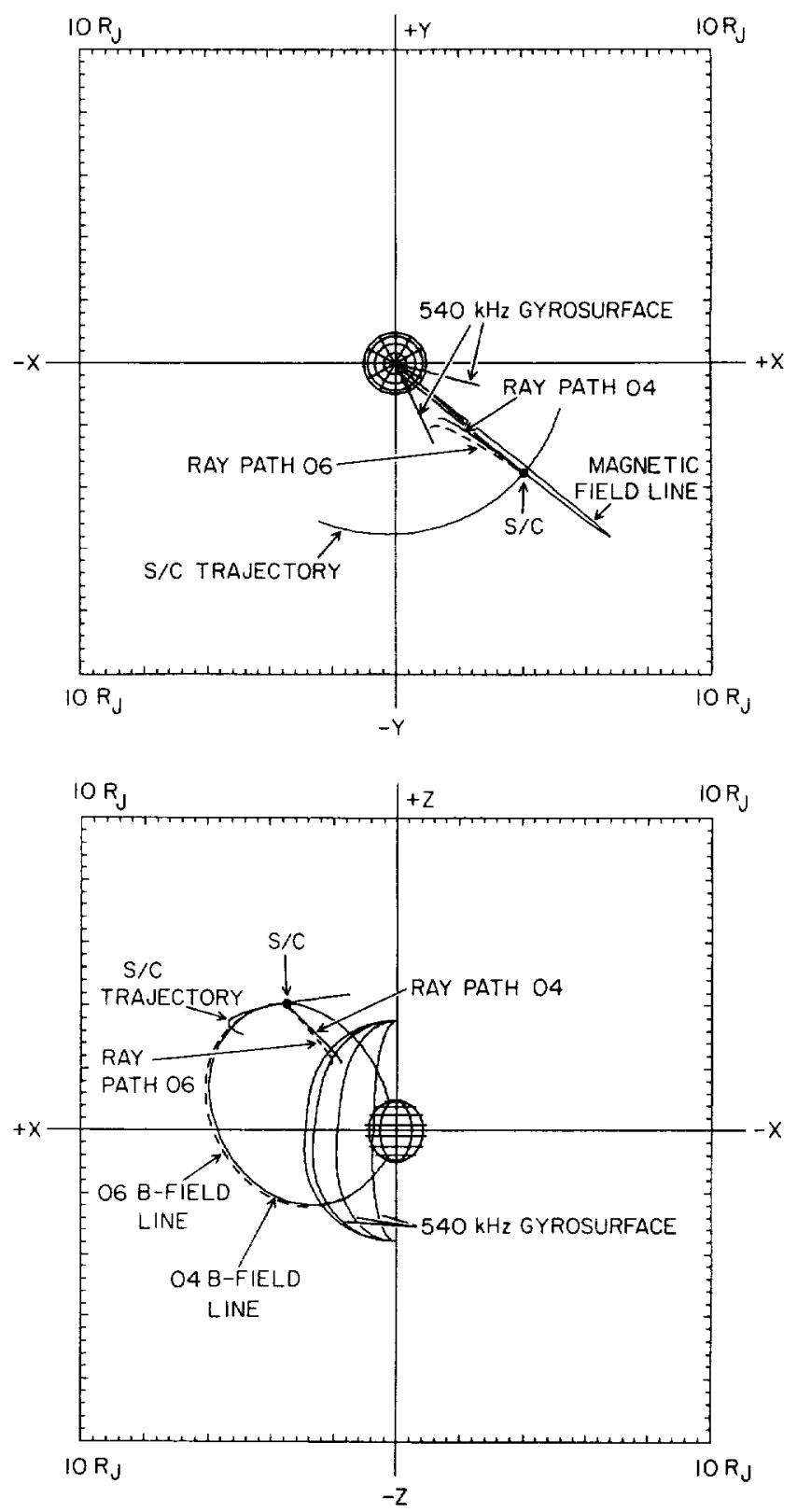

Figure 5. (a) Top and (b) three-dimensional views of ray paths for waves launched at $f=540 \mathrm{kHz}$ from the event 2 satellite position and in the same direction relative to the magnetic field. In one case the O6 magnetic field model was used and in the other case the $\mathrm{O} 4$ magnetic field model was employed. This plot indicates differences in ray path due solely to the effects of the magnetic field model. 
to modify the initial wave direction angles by more than $15^{\circ}$ in order for this to occur, far outside the error estimate.

For event 2 we investigated the effects of modifications in the plasma density (by comparing runs with and without a plasma torus) and modification of the magnetic field (comparing the $\mathrm{O} 6$ to the $\mathrm{O} 4$ model). However, in all cases it was necessary to modify the ray direction angles, $\Delta \mathbf{k}$, by significantly more than the $4^{\circ}$ estimated error limit in order for the ray to intercept $f_{\mathrm{RX}}$, a necessary condition for a source region.

Our results indicate that refractive effects due to the Io plasma torus and the magnetic ficld are significant for HOM propagation. As seen in Figures 2 and 3, rays can be significantly refracted out of the meridian plane, indicating source locations that are at longitudes that differ by perhaps tens of degrees from the spacecraft meridian plane. Results for event 1 (Table 2) suggest modest refraction out of the meridian plane from source positions located along magnetic field lines with $L<7$ and require initial wave directions at the spacecraft that differ by $\leqslant 3^{\circ}$ from the observations. For event 2 , however, we find that we must modify the launch directions at the spacecraft by $>15^{\circ}$ (over 3 times the estimated uncertainty) in order for the ray to intercept a possible source region. We suggest that differences between the Io plasma density and magnetic field models compared to actual conditions at the time of the Ulysses flyby may account for part of the magnitude of $\Delta k$ obtained in our results for event 2 , which occurred about 8 hours after event 1 . Temporal effects and azimuthal inhomogeneities in the plasma density of the Io torus with magnitudes reaching a factor of 2 or more have been observed for a number of years [of. Schneider et al., 1989; Schneider and Trauger, 1995; Desch et al., 1994; Kaiser et al., 1996]. Such inhomogencities have not been incorporated in our study which assumes azimuthal symmetry. As noted carlier, we have modified the entire Io torus density by factors ranging from 0 to 5 with unsatisfactory results. Faraday rotation measurements [Warwick and Dulk, 1964] and more recent polarization studies [Dulk et al., 1994] have suggested that the electron density close to Jupiter may be quite low $\left(<1 \mathrm{~cm}^{3}\right)$. However, we have modified the inner plasmaspheric density $(r<4 R$ ) of the Divine-Garrett model also by factors ranging from 0 to 5 (results not shown), but were unable to significantly decrease $\Delta \mathbf{k}$ for event 2. All of these modifications maintained azimuthal symmetry, however. Large plasma clouds rotating within the torus, for instance, have not been modeled. Anomalies in the magnetic field model might also explain our results for event 2. Connerney [1993] discusses in some detail the possibility of $B$ field anomalies, especially at low altitudes where in situ measurements do not exist.

These results illustrate the difficulty of accurately locating HOM radio sources in the Jovian magnetosphere. Even though in this analysis we have for the first time incorporated rather precise information on the incident direction of the radiation at the spacecraft, tracing these rays back to the source region requires an accurate, time-dependent and spatially inhomogeneous Jovian magnetospheric model, which is not realized at present. We therefore have to be content with an approximate HOM source location determination.

It is significant that the largest value of $L$ obtained in our ray-tracing analysis was $L_{\text {max }}=7$, which is less than the $L$, shell range of HOM source regions suggested by Ladreiter and Leblanc [1990a] of $15<L<20$ (auroral zone sources). The latter study was performed without knowledge of the observed wave directions that we have incorporated in our study.

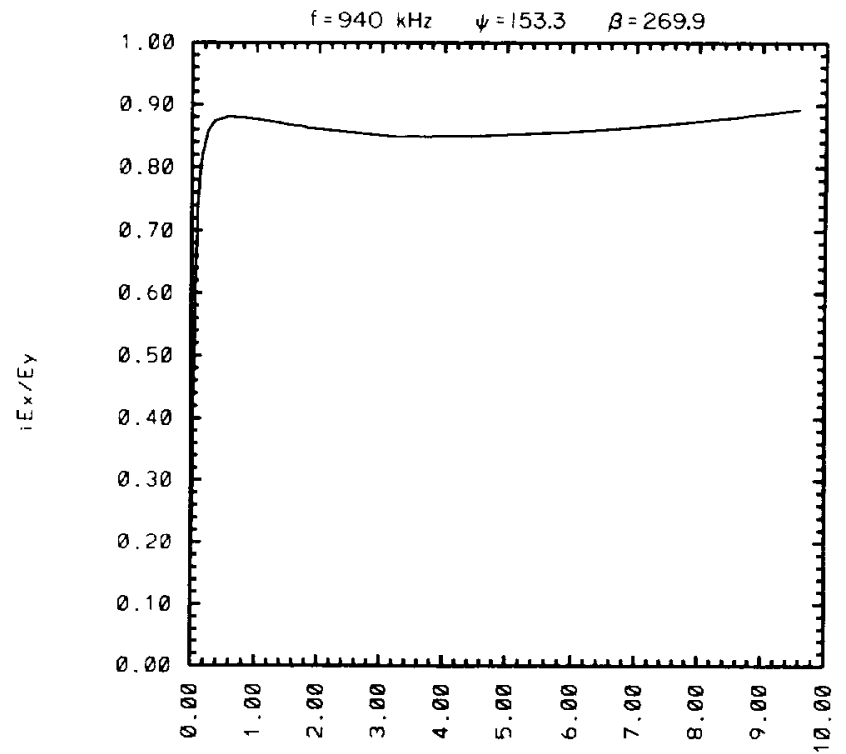

Figure 6. Polarization versus distance from the source $(R$, for a typical ray path.

\section{Conclusions}

We have performed a ray tracing analysis of Jovian HOM emission. Because of the unique direction-finding capability of the URAP instrument on board Ulysses, we have for the first time to our knowledge used measured wave directions at a spacecraft to initialize a ray tracing procedure. The calculations were presented for two distinct spacecraft locations in the Jovian magnetosphere, where HOM emission was observed (events 1 and 2), and for several frequencies. The ray tracing calculations were performed using a model of the Io torus [Divine and Garrett, 1983], and two different Jovian magnetic field models (O4 and $\mathrm{O} 6$ ). It was found that significant wave refraction occurred for the $\mathrm{HOM}$ cmission due both to the lo torus, and the Jovian magnetic field model, however, calculated source locations for the HOM all occur on Jovian field lines with $3 \leq L<7$ in agreement with the results of Reiner et al. [1993a]. The ray tracing results for event 2 strongly imply either a temporal or spatial asymmetry or anomaly in either the plasma density or magnetic field structure.

Finally, calculated polarization of the emission is $\ll 1$ near the source region, and $\sim 1$ near the spacecraft as observed. This result is due, in part, to the wave normal angle dependence of the index of refraction.

Acknowledgments. We are grateful to D. A. Gurnett and N. Schneider for many useful comments regarding this work and to K. Kurth and JooHee Chung for formatting the manuscript. This work wals supported by contract 958779 with the Jet Propulsion Laboratory.

The Editor thanks Y. Leblanc and T. D. Carr for their assistance in evaluating this paper.

\section{References}

Acuna, M. H., and N. F. Ness, The main magnelic field of Jupiter. $J$ (ieophys. Res., 81, 2917, 1976.

Bagenal, F., Empirical model of the Io plasma torus: Voyager measurements, J. Cocophys. Res., 99, $11,043,1994$.

Batgenal, F., and J. D. Sullivan, Direct plasma measurements in the Io torus and inner magnetosphere of Jupiter, J. (iecophys. Re's., \&6, 8447, 1981 . 
Bagenal, F., R. L. McNutt Jr., J. W. Belcher, H. S. Bridge, and J. D. Sullivan, Revised ion temperatures for Voyager plasma measurements in the Io plasma torus, J. Geophys. Res., 90, 1755, 1985.

Calvert. W., The source location of certain decametric radio emissions, J. Geophys. Res., 88, 6165, 1983.

Carr, T. D., M. D. Desch, and J. K. Alexander, Phenomenology of magnetospheric radio emissions, in Physics of the Jovian Magnetosphere, edited by A. J. Dessler, p. 226, Cambridge Univ. Press, New York, 1983.

Connerney, J. E. P., Magnetic fields of the outer planets, J. Geophys. Re's., 98, 18,659, 1993.

Desch, M. D., W. M. Farrell, and M. L. Kaiser, Asymmetries in the to plasma torus, J. Geophys. Res. 99, 17,205, 1994.

Divine, N., and H. B. Garrett, Charged particle distributions in Jupiter's magnetosphere, J. Geophys. Res., 88, 6889, 1983.

Dulk, G. A., Y. Leblanc, and A. Lecacheux, The complete polarization state of Io-related radio storms from Jupiter: A statistical study, Astron. Astrophys., 286, 683, 1994.

Hasclgrove, J., Ray theory and a new method of ray tracking. in Report of Conference on the Physics of the Ionosphere, pp. 355, London Phys. Suc., London, 1955.

Kaiser, M. L., M. D. Desch, and M. E. Brown, Evidence for an Io plasma torus influence on high-latitude Jovian radio emission, $J$. Geophys. Res., 101, 13, 1996.

Ladreiter, H. P., and Y. Leblanc, Jovian hectometric radiation: Beaming, source extension, and solar wind control, Astron. Astrophys., 226, $297,1989$.

Ladreiter, H. P., and Y. Leblanc, Source location of the Jovian hectometric radiation via ray-tracing technique, J. Geophys. Res., 95. $6423,1990 \mathrm{a}$

Ladreiter, H. P., and Y. Leblanc, Modeling of the Jovian hectometric radiation: A three-dimensional study, Ann. Geophys., 8, 477, 1990)

Ladreiter, H. P., P. Zarka, and A. Lecacheux, Direction finding study of Jovian hectometric and broadband kilometric radio emissions: Evidence for their auroral origin, Planet. Space Sci., 42, 919, 1994.

Manning, R., and J. Fainberg, A new method of measuring radio source parameters of a partially polarized distributed source from spacecraft observations, Space Sci. Instrum., 5, 161, 1980.

Melrose, D. B., and G. A. Dulk, Electron cyclotron maser emission at oblique angles, Planet. Space Sci., 41, 5, 1993.

Menietti, J. D., J. L. Green, N. F. Six, and S. Gulkis, Ray tracing of Jovian decametric radiation from southern and northern hemisphere sources: Comparison with Voyager observations. J. Geophys. Res., 92, 27, 1987.

Reiner, M. J., J. Fainherg, and R. G. Stone, Source characteristics and locations of hectometric radio emissions from the Northern Jovian hemisphere, Geophys. Res. Lett., 20, 321, 1993a.

Reiner, M. J., J. Fainberg, and R. G. Stone, Source characteristics of Jovian hectometric radio emissions, J. Geophys. Res., 98, 18,767, 1993 b.

Schneider, N. M., and J. Spencer, Io and Jupiter: The volcanomagnetosphere connection, Eos Trans., AGU, 73, 55, 1991.

Schneider, N. M., and J. T. Trauger, The structure of the Io torus, Astrophys. J., 450, 450, 1995.

Schneider, N. M. W. H. Smyth, and M. A. McGrath, Io's atmosphere and neutral clouds, in Time Variable Phenomena in the Jovian System, edited by M. J. S. Belton, R. A. West, and J. Rahe, NASA Spec. Publ.. SP-494, 75, 1989.

Stix, T. H., The Theory of Plasma Waves, McGraw-Hill, New York. 1962.

Warwick, J. W., and G. A. Dulk, Faraday rotation on decametric radio emissions from Jupiter, Science, 145, 380, 1964.

J. D. Menietti, Department of Physies and Astronomy, Van Allen Hall, University of lowa, lowa City, IA 52242. (e-mail: jdm(a space.physics tuiowa.tedu)

M. J. Reiner, Hughes STX, 4400) Forbes Blvd., Lanham, MD 20706.

(Received June 21, 1996: revised July 31, 1996; accepted July $31,1996$. 